\title{
Extraction of conjugate main-stream structures from a complex network flow
}

\author{
Koutarou Tamura, ${ }^{1}$ Hideki Takayasu, ${ }^{1,2,3}$ and Misako Takayasu ${ }^{1}$ \\ ${ }^{1}$ Department of Computational Intelligence and Systems Science, Interdisciplinary Graduate School of Science and Engineering, \\ Tokyo Institute of Technology, 4259-G3-52 Nagatsuta-cho, Midori-ku, Yokohama 226-8503, Japan \\ ${ }^{2}$ Sony Computer Science Laboratories, 3-14-13 Higashigotanda, Shinagawa-ku, Tokyo 141-0022, Japan \\ ${ }^{3}$ Meiji Institute for Advanced Study of Mathematical Sciences, Meiji University, 4-21-1 Nakano, Nakano-ku, Tokyo 164-8525, Japan
}

(Received 13 January 2015; published 30 April 2015)

\begin{abstract}
We introduce a method to extract main-stream structures for a given complex network flow by trimming less effective links. As the resulting main streams generally have an almost loopless treelike structure, we can define the stream basin size for each node, which characterizes the importance of the node with regard to the flow. As a real-world example, we apply this method to an interfirm trading network, both for the money flow and its conjugate - the material or service flow—confirming that both basin size distributions follow a similar power law that differs significantly from the basin size distributions of rivers in nature. We theoretically analyze the process of trimming and derive a consistent statistical formulation between the original link number and the basin size.
\end{abstract}

DOI: 10.1103/PhysRevE.91.042815

PACS number(s): 89.75.Hc, 92.40.Cy, 89.75.Da, 02.50.-r

\section{INTRODUCTION}

The transport phenomena of complex network systems have attracted the attention of physicists to a greater extent in comparison with well-known transport properties in thermal equilibrium [1-7]. Researchers of complex systems have reported the nonlinearity of local interactions [8-11] and investigated the flow for nontrivial network structures, where the basic equilibrium concept of detailed balance was no longer a natural assumption.

We consider the dendritic network flows of rivers in nature as typical examples of a nonequilibrium network flow. Scheidegger focused on river structures and proposed a simple random coagulation model for the river network structure [12]. Inaoka and Takayasu introduced an erosion dynamics model and studied the geometrical structures of the flow path, deriving a power-law distribution for the flow intensity [13]. As forerunners in the application of network analysis for river flow analysis, Horton [14], Strahler [15,16], and Shreve [17] recognized the river structure as a binary branch and defined a degree representing the statistical characteristics of the flow path. Using these ideas, the structures of flow systems such as blood vessels [18,19] and leaf vessels [20] are evaluated and categorized. The quantities of the degree and branching ratio are theoretically known to relate to a fractal dimension of the geometrical structure of the flow path [21-23].

Recently, studies of transport in complex systems have extended to social phenomena. For example, human transport between nations [8] and cities [9,10], i.e., immigration and trafficking, are studied in the framework of general transport phenomena. Additionally, transactions between firms [11] and trades between nations are considered from the viewpoint of money flow [24]. In particular, the trading network data of business firms are investigated intensively as typical real-world examples of scale-free networks; a node denotes a firm, and a

Published by the American Physical Society under the terms of the Creative Commons Attribution 3.0 License. Further distribution of this work must maintain attribution to the author(s) and the published article's title, journal citation, and DOI. directed link denotes the money flow from a buyer to a seller [7,11,25-27]. Watanabe et al. proposed a model for money transport on an interfirm trading network to explain empirical properties such as the power-law distribution of sales [7]. The authors reported a fundamental nonlinear property of money flow by analyzing transactional data and showing that the transaction volume between firms is determined by the product of the asymmetric fractional power of the sales of the trading firms [11]. This type of interaction is known in various fields as a "gravity interaction," and it is observed in international trade [24,28], immigration [8], and traffic between cities [9,10].

There have been studies of methods for extracting important substructures in complex networks. Mantegna et al. applied the minimum spanning tree for correlation among the market prices of stocks, deriving a loopless tree structure [29]. Glattfelder proposed a method to extract a backbone structure in an ownership network whose nodes and weighted links indicated companies and shareholding relations [30]. Anand et al. applied the maximum entropy method for an interbank network to estimate counterparty exposures, and they proposed a method to reveal an important structure with minimum density by examining the most probable links [31].

In this paper, we focus on the network structure of money flow and its conjugate-material or service flow-among business firms comprising 3.8 million links. First, in Sec. II, we confirm some basic properties of interfirm trading network data. In Sec. III, we propose a method to trim unimportant links and extract a flow basin structure. We show that the basin size distributions follow nontrivial power laws representing the topological properties of the structure. In Sec. IV, we theoretically analyze the statistical properties of our method and reveal the relation between the original network and its trimmed basin network. In Sec. V, we discuss the statistical properties of basin structures and their practical meaning using the automobile industry as an example. The final section presents a conclusion.

\section{DATA ANALYSIS}

The network data analyzed in this study, provided by Teikoku Data Bank, correspond to a Japanese interfirm trading 
network comprising 627262 nodes and 3844684 links for the year 2011 [11]. The data include partial information about the transaction volumes for 40159 links.

As the most fundamental property of interfirm trading networks, it is reported that the degree distributions are symmetric with regard to in-degrees and out-degrees and obey power laws with power exponents of $1.45[11,25]$. The network structure has a weak negative correlation of links, and the distribution of firms' sales and employees obeys power laws with exponents of 1.0 and 1.35 , respectively $[11,25]$. These topological and nontopological quantities correlate well with each other. Watanabe et al. reported that the network structure, i.e., the number of trades $k$, of firm $m$ is correlated with the sales $S$ of firm $m$ according to the power-law scaling function [32]:

$$
S_{m} \propto k_{m}^{1.3}
$$

A transaction represents the interaction between a buyer $i$ and a supplier $j$. The intensity of an interaction, that is, the transaction volume $f_{i j}$, is estimated according to the product of the fractional powers of both sales with different exponents [11]:

$$
f_{i j} \propto A_{i j} S_{i}^{\epsilon} S_{j}^{\delta}
$$

where $A_{i j}$ is the adjacency matrix element of an interfirm trading network, which is 1 if there exists a money flow from firm $i$ to $j$, otherwise it is 0 . The typical values are $\epsilon=0.9$ and $\delta=0.3$, which differ significantly from those in the case of thermal diffusion on networks, $\epsilon=1$ and $\delta=0$, including the method of PAGERANK [2]. The authors focused on this nonlinear interaction and proposed a practical model to estimate the interfirm systemic risk [33]. We can observe the inequality of flows - a basic aspect of the nonlinear flowas shown in Fig. 1(a). For each node having transactional information, the magnitudes of the outflows are ranked in descending order, and their shares are estimated as a function
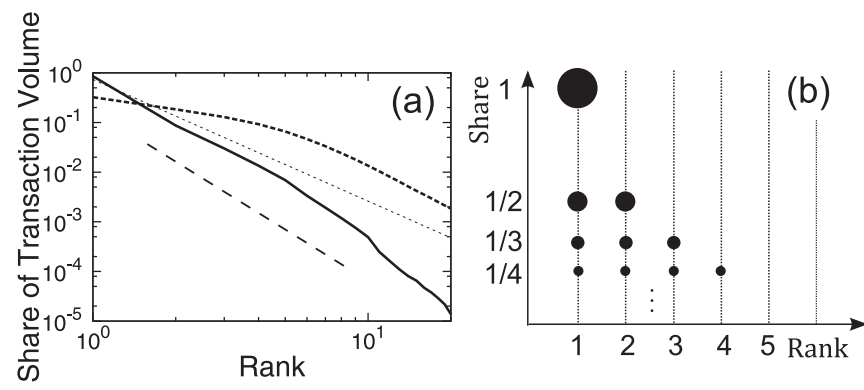

FIG. 1. Flow shares as a function of rank, estimated from transactional data for 31850 companies. (a) The solid line denotes the rank-share relation of real transactional data. The dashed straight line along the former line denotes a power function with exponent -3.5 . The thick dotted line denotes the theoretical share of equipartition flow, derived by Eq. (3), with $P(k)$ assigned to the real degree distribution of an interfirm trading network. The thin dotted line denotes the power law derived by Eq. (3) with $P(k) \sim k^{-2.45}$. (b) Schematic view of calculating shares in each rank in the case of equipartition transport. The share defined on an out-link is plotted with a rank of the out-link in every case. The size of the circle denotes weight. of the order, where the share is defined according to the transaction volume and normalized by the total volume of the node. As shown in this figure, the share of the top rank is outstanding, while the others provide a far smaller contribution. The share of the top rank is approximately three times larger than that of the second one. Consequently, the form of the overall function is empirically approximated by a power function with the exponent -3.5 .

The thick dotted line in Fig. 1(a) indicates the theoretical share values in the case of thermal transport $\delta=0$, that is, the equipartition-type transport. This rank-share relation can be estimated as follows. A node with out-degree $k$ has $1 / k$ share of the flow for connecting nodes with rank 1 to $k$, described as $k$ circles plotted horizontally in Fig. 1(b), and its weight is proportional to $P(k)$, i.e., the out-degree probability density of a given network. The theoretical share value of rank $i, S(i)$, in the case of equipartition is obtained by the sum of all circles lined in Rank $i$ divided by the sum of all circles for all ranks. Precisely, it is given as follows:

$$
S(i)=\frac{\sum_{k=i}^{\infty} \frac{1}{k} P(k)}{\sum_{i=1}^{\infty} \sum_{k=i}^{\infty} \frac{1}{k} P(k)} .
$$

We can approximate $P(k)$ as a power-law distribution, $k^{-(1+\alpha)}$, with $\alpha=1.45$, and the summation in Eq. (3) can be evaluated using an integral. Then, we obtain the theoretical line shown in Fig. 1(a). For a very large rank number $i$, we have the following rank-share relation of equipartition-type transport:

$$
S(i)=\frac{1}{\zeta(1+\alpha)} i^{-(1+\alpha)},
$$

where $\zeta(1+\alpha)$ is the Riemann-zeta function of the parameter $\alpha$ for the normalized constant $\sum_{i=1}^{\infty} S(i)=1$. It should be noted that the rank-share relation obtained by the real data is significantly different from the theoretical equipartition line. This type of unequal partition among the flows is a major characteristic of a gravity-type flow, which we expect to occur broadly in social phenomena.

\section{EXTRACTION OF THE MAIN STREAM}

To extract the main flow structure for any given complex directed network without quantitative flow data, we introduce a simple method for trimming the links from each node, except for the link to the destination with the largest number of in-links, as shown in Figs. 2(a) and 2(b). This procedure corresponds to the limit of large $\delta$ in Eq. (2) with the scaling relation of Eq. (1), as all flow emitted from a node goes to the destination node with the largest number of in-degree in such a case. We confirmed from the business firm data that the largest in-degree neighbor node actually receives the largest flow among rival neighbors with an accuracy of about $74 \%$, therefore this approximation is considered to be reasonable for networks with limited information about flow. If there are more than two destinations having the same largest number of in-links, one link is chosen randomly. As each node has at most one out-link by this trimming method, the trimmed network has an almost treelike structure with at most one loop path for each connecting cluster of links. We call this extracted subnetwork the "basin network." 

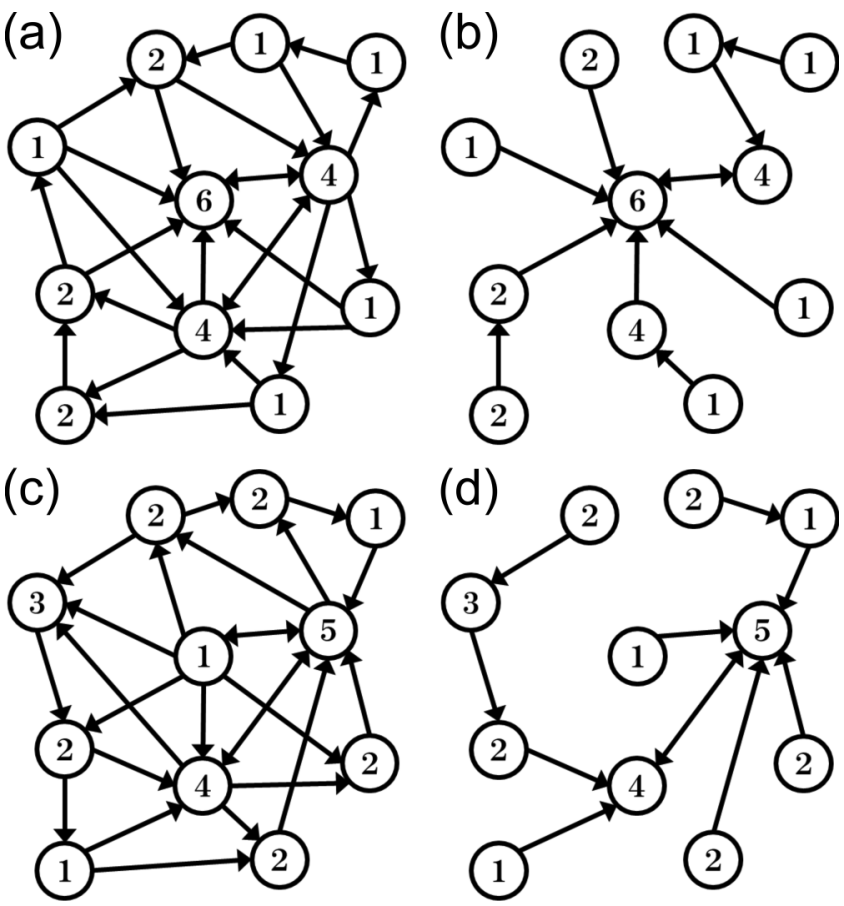

FIG. 2. Examples of trimming and basin structures. The number in a node denotes the number of its in-links. Original directed network (a) and its basin structure (b). Network with reversed direction (c) and its basin structure (d).

The flow of a network generally has duality. For example, in the case of an interfirm trading network, the direction of the link is usually defined by the direction of the money flow, and by reverting the direction, we obtain the conjugate flow: that of materials or services. Figure 2(c) shows an example of the conjugate network of Fig. 2(a), in which all the directions of the links are reversed, and Fig. 2(d) shows the basin structure of this conjugate network. Comparing Figs. 2(b) and 2(d), we find that these dual basin networks are significantly different.

We apply the aforementioned trimming method to the business firm network data comprising 0.6 million nodes. As shown in Figs. 3(a) and 3(b), the degree distributions of basin structures for the money flow network and the material or service flow network follow very similar power laws, with exponents of 1.0, whereas the original degree distributions follow power laws with exponents of 1.45 for both the money flow and the material or service flow. It is known from these figures that more than $90 \%$ of the nodes have only one link in the trimmed basin networks in both the money flow and material or service flow.
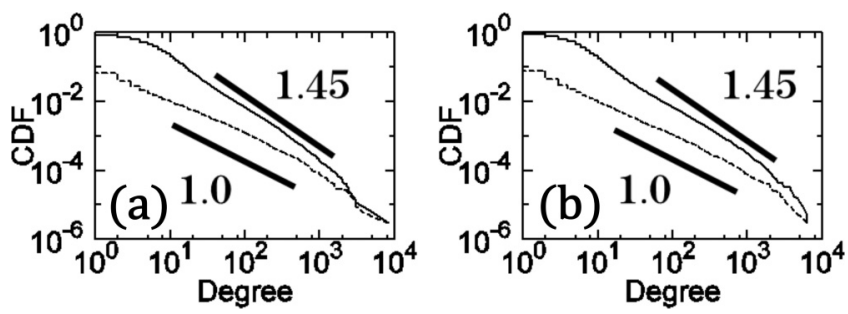

FIG. 3. Cumulative distributions of degree and basin degree. Part (a) shows money flow and (b) shows material or service flow.
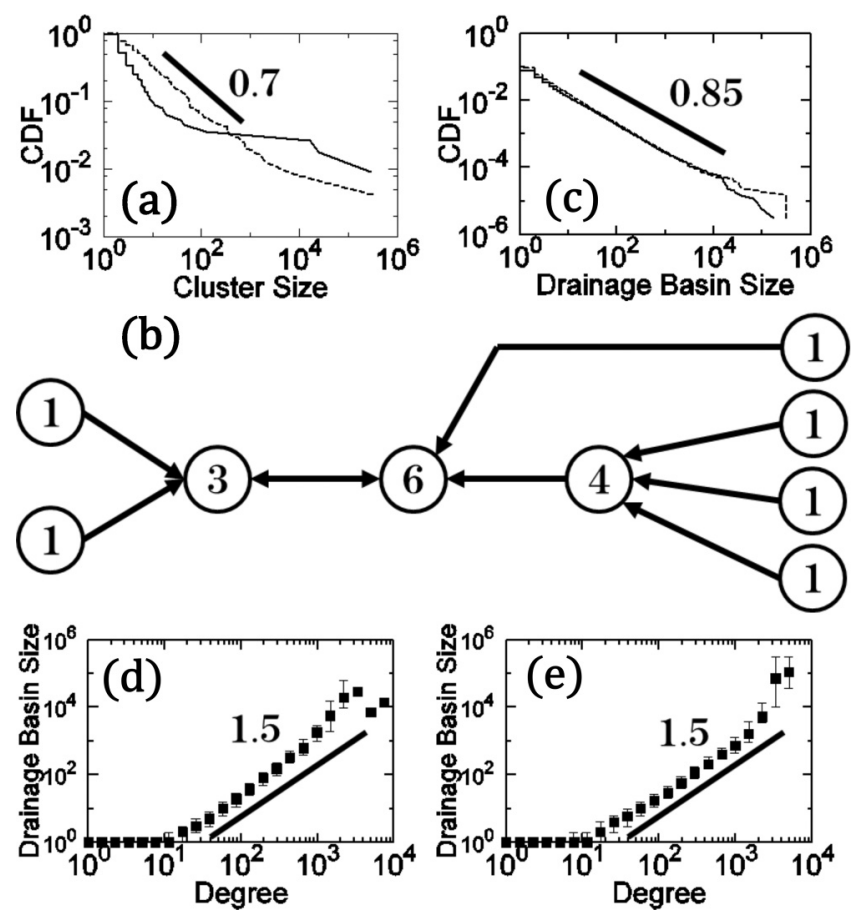

FIG. 4. Basic properties of the resulting network. (a) Cumulative distribution of the cluster size for money flow (solid) and material or service flow (dotted). (b) Schematic view of the drainage basin size of a node on a treelike network. The number in a node denotes the value of the drainage basin size defined on the specified node. (c) Cumulative distribution of the drainage basin size for money flow (solid) and material or service flow (dotted). Relation between drainage basin size and degree on an initial network; money flow (d) and material or service flow (e). Error bars indicate the quartiles.

Globally, the resulting basin network comprises clusters of a treelike structure. Let us consider the basin network structure. The basin structure obtained from the largest component of a strongly connected network loses its global connectivity. The number and size of the separated clusters are characterized by the asymmetric distributions, as shown in Fig. 4(a). As both distributions have long tails in their log-log plots, the mean size of the clusters is not an appropriate statistical quantity to understand the topology. Paying attention to their almost loopless structures, the upstream and downstream of the flow in the basin network becomes explicit, except for the loops. We can define the drainage basin size for each node by avoiding the double count from the loop, following the standard technique used in the study of rivers [13]. As shown in Fig. 4(b), the drainage basin size of a node is given by the sum of the numbers of all nodes located in the upper stream, including itself. This definition suggests that the drainage basin size of a certain node contains information about the structure of the upper stream and can thus be regarded as an index to characterize the network structure.

The drainage basin sizes for the basin structure embedded in the real interfirm trading network are calculated for all nodes. For nodes having loop links, no contribution from the loops is considered. Figure 4(c) shows that the drainage basin size distributions for the money flow and material or service flow are quite symmetric, following an identical power law with an exponent 0.85 over the entire range. This exponent is an 
important number characterizing the network in the study of river-structure analysis. In particular, river patterns in nature and simulated river patterns created by water erosion are known to follow power-law distributions of the drainage basin area with an exponent of 0.4 [13], which is significantly smaller than the aforementioned value for the money and material or service flow networks. This remarkable difference implies that the structure of the basin for the money flow and that for the river flow are very different, although they are both treelike.

Interestingly, the basin network is an embedded structure in the original complex network, and a comparison with the initial network structure can yield further understanding of the initial network structure. Figures 4(d) and 4(e) show the relation between the degrees of the initial network and the size of the drainage basin in the trimmed network. The behavior changes drastically around $k=20$; that is, the nodes below $k=20$ are likely located at the uppermost edges with drainage basin size unity in the basin network. For nodes with original degrees larger than 20, the corresponding drainage basin size increases as a power function on average; i.e., such firms tend to have at least one supportive business partner in the upper stream.

\section{THEORETICAL ANALYSIS}

We analyze the statistical properties of the basin networks. According to the trimming rule whereby only the out-link connected to the largest in-degree node survives, a link to a node with a small in-degree is likely to be removed in a statistical sense. We consider the change in the in-degrees before and after the trimming process.

Let us assume an uncorrelated network with the degree distributions $P_{\text {in }}(k)$ and $P_{\text {out }}\left(k^{\prime}\right)$ for in-degrees and out-degrees, respectively. In this network, the probability that a source node of a directed link in the network belongs to out-degree $k^{\prime}$ is determined according to the degree distribution of the neighbors' out-degree, as follows:

$$
f_{s}\left(k^{\prime}\right)=k^{\prime} P_{\text {out }}\left(k^{\prime}\right) /\left\langle k^{\prime}\right\rangle
$$

Similarly, the probability that a sink node of a directed link in the network belongs to in-degree $k$ is given as

$$
f_{t}(k)=k P_{\text {in }}(k) /\langle k\rangle \text {. }
$$

Combining these relations, the joint probability $P\left(k^{\prime}, k\right)$ that a link has a source node of out-degree $k^{\prime}$ and also has a sink node of in-degree $k$ is calculated as

$$
P\left(k^{\prime}, k\right)=f_{s}\left(k^{\prime}\right) f_{t}(k) \text {. }
$$

We estimate the number of such links as $L P\left(k^{\prime}, k\right)$, where $L$ is the total number of links in the network.

Next, we focus on a node that has $k^{\prime}$ out-links. We evaluate the probability that the in-degree $k$ is the largest in-degree among the $k^{\prime}$ neighbor nodes, that is, the probability that the $k^{\prime}-1$ nodes have less than $k$ links. This probability is given as $[F(k)]^{k^{\prime}-1}$, where $F(k)=\int_{1}^{k} f_{t}(x) d x$. Using this formula, we estimate the number of links from the node having $k^{\prime}$ out-links to the node having $k$ in-links as $L P\left(k^{\prime}, k\right)[F(k)]^{k^{\prime}-1}$, and the number of links to the node having $k$ in-links is estimated as

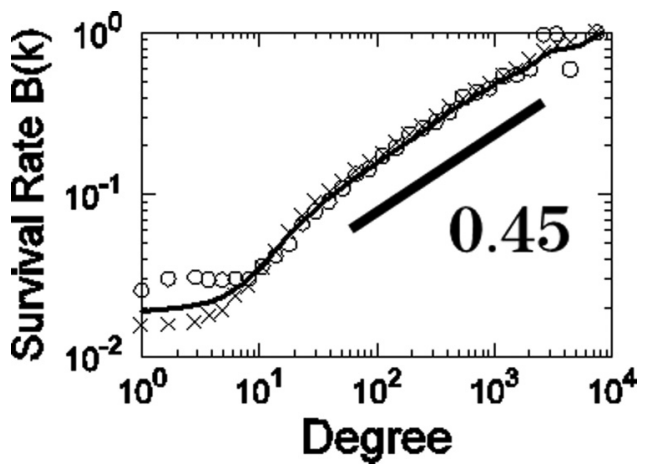

FIG. 5. Survival rate $B(k)$ as a function of initial degree. Circles and crosses indicate the measured values of a real interfirm trading network and its randomly rewired network, respectively. The solid line denotes the theoretical value according to Eq. (10), using the real distributions $P_{\text {out }}\left(k^{\prime}\right)$ and $P_{\text {in }}(k)$.

follows:

$\sum_{k^{\prime}=1}^{k_{\max }^{\prime}} L P\left(k^{\prime}, k\right)[F(k)]^{k^{\prime}-1}=L f_{t}(k) \sum_{k^{\prime}=1}^{k_{\max }^{\prime}} f_{s}\left(k^{\prime}\right)[F(k)]^{k^{\prime}-1}$.

Similarly, the number of links to the node having $k$ in-links in the original network is also obtained as $L f_{t}(k)$. The survival rate $B(k)$ is defined for a node of in-degree $k$ by the ratio of the number of nontrimmed links divided by $k$, and it is evaluated as follows:

$$
\begin{aligned}
B(k) & =\sum_{k^{\prime}=1}^{k_{\max }^{\prime}} f_{s}\left(k^{\prime}\right)[F(k)]^{k^{\prime}-1} \\
& =\frac{G_{X}^{\prime}[F(k)]}{G_{X}^{\prime}(1)}
\end{aligned}
$$

where $G(x)$ is the generating function, defined by the outdegree distribution as

$$
G(x)=\sum_{k^{\prime}=1}^{k_{\max }^{\prime}} P_{\mathrm{out}}\left(k^{\prime}\right) x^{k^{\prime}}
$$

Assigning the observed $f_{s}\left(k^{\prime}\right)$ and $F(k)$ numerically, we verify that the above derivation agrees with the observation, as shown in Fig. 5. This function $B(k)$ quantitatively characterizes the relation between the original network and the basin network.

Conversely, by viewing the link removal as a stochastic process, the probability $p_{k}(\tilde{k})$ that the node belonging to the indegree $k$ in the original network becomes a node with in-degree $\tilde{k}$ in the basin network is given by the following binomial distribution with the survival rate $B(k)$ :

$$
p_{k}(\tilde{k})={ }_{k} C_{\tilde{k}}[1-B(k)]^{k-\tilde{k}} B(k)^{\tilde{k}} .
$$

Using this equation, we estimate the basin network's degree distribution $Q(\tilde{k})$ as

$$
Q(\tilde{k})=\sum_{k} P_{\text {in }}(k) p_{k}(\tilde{k}) .
$$

Taking the average over the degree, $\tilde{k}(k)=\sum_{\tilde{k}} \tilde{k} p_{k}(\tilde{k})=k B(k)$, the trimming process transforms the degree distribution from 
$P_{\text {in }}(k)$ to $Q(\tilde{k})$ by changing the variable $k$ into $\tilde{k}$.

$$
Q(\tilde{k}) d \tilde{k}=P_{\text {in }}(k) d k \Leftrightarrow Q(\tilde{k})=P_{\text {in }}(k) \frac{1}{(d \tilde{k} / d k)} .
$$

In the special case in which the nodes having $k$ in-links change their in-degrees to $\tilde{k}(k)=k B(k) \sim k^{1+\gamma}$ in the basin network, the degree distribution $Q(\tilde{k})$ fulfills the following scaling relation for any initial degree distribution $P_{\text {in }}(k)$ :

$$
Q(\tilde{k}) \sim P_{\text {in }}\left(\tilde{k}^{\frac{1}{1+\gamma}}\right) \tilde{k}^{\frac{-\gamma}{1+\gamma}} .
$$

Let $P_{\text {in }}(k)$ be a power-law distribution $k^{-(1+\alpha)}$. Assuming a scaling relation, $Q(\tilde{k}) \sim k^{-(1+\beta)}$, the following relation is obtained:

$$
\beta=\frac{\alpha}{1+\gamma} .
$$

Combining the results of the value $\gamma=0.45$ obtained from Fig. 5 and the power exponent of the real degree distribution for $P_{\text {in }}(k), \alpha=1.45$, we estimate the power exponent of the degree distribution of the basin network as 1 , which is consistent with Fig. 3.

On the contrary, if we assume the degree distribution as $Q(\tilde{k}) \sim \tilde{k}^{-2}$, the survival rate $B(k)$ is estimated to follow a power function with exponent 0.45 . It is straightforward to apply the above formulation to the conjugate network defined by reverting the direction of all links.

According to the former discussion, we obtain two theoretical relations: the relation between the initial degree distribution and the survival rate, described by Eq. (10), and the relation among the three power exponents, represented by Eq. (16). To confirm the validity of these theoretical results, we analyze artificially created scale-free networks. Using the configuration method [34], we prepare three networks whose degree distributions are characterized by the same power exponent $\alpha=1.45$; however, their averaged degrees are different: $\langle k\rangle=1,5$, and 10. Figures 6(a) and 6(b) show the distribution of the basin degree and the survival rates in the case of $\langle k\rangle=1$. The theoretical line in Fig. 6(b) fits well with the measured curve, and the relation among the three power exponents derived in Eq. (16) is satisfied. As higher degree nodes have a higher probability of escaping from our trimming process, the value of $\gamma$ is expected to be positive in general. Therefore, $\alpha$ should be larger than $\beta$ in this case.

Figures 6(c) and 6(d) show the results for the network with the average degree $\langle k\rangle=5$, which is approximately equal to that in the real interfirm trading network. The basin degree distribution and survival rates are very similar to the results obtained for the real interfirm trading network, Figs. 3 and 5, and the exponents satisfy Eq. (16).

In the case of the network with the larger averaged links, where $\langle k\rangle=10$, the estimated power exponent of the basin size distribution is 0.7 , which is significantly smaller than the case of a real network, as shown in Fig. 6(e). Figure 6(f) demonstrates that the function $B(k)$ deviates clearly from the results of the real case shown in Fig. 5. These results indicate that the parameter of the averaged link number plays an important role in the construction of drainage basins. Even in this case, the estimated exponents $\beta=0.7$ and $\gamma=1.1$ satisfy Eq. (16) with $\alpha=1.45$.
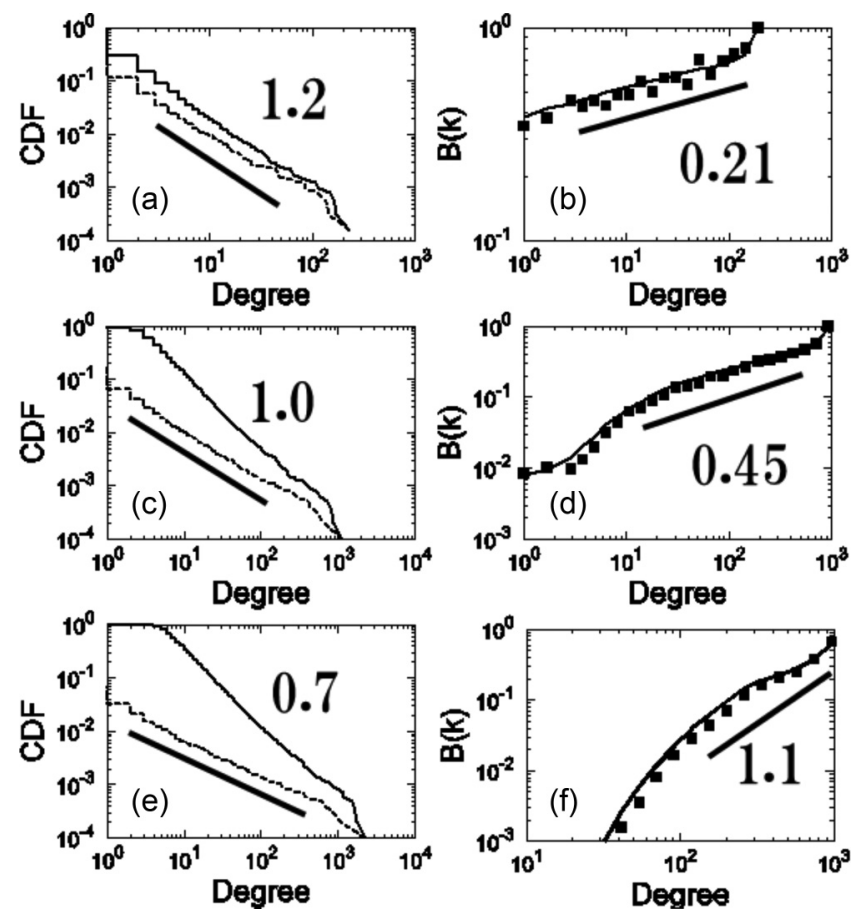

FIG. 6. Numerical results of the trimming process for scalefree networks whose power exponent of 1.45 is created using the configuration model. The direction is defined on undirected links with a probability of 0.5 . The pairs of results-(a) and (b), (c) and (d), and (e) and (f) - are produced under the same initial conditions, with different average degrees: 1,5 , and 10, respectively. The figures in the left column show the degree distributions of a generated network (solid line) and its basin network (dotted line). The figures in the right column show the survival rates of the corresponding cases.

\section{DISCUSSION}

The trimming method proposed in this paper is statistically equivalent to a process of random removal of links satisfying the survival rate, $B(k)$, given by Eq. (10). In particular, in the case in which the survival rate $B(k)$ is a constant $f(0<f<1)$, a link is removed randomly, independent of the degree of the connected node. This removal process we proposed can be interpreted as a type of bond-percolation process of the network that removes links according to the node's degree.

As another aspect of the aforementioned theory, we can transform the structure of any scale-free network with a power exponent of degree distribution $\beta$ to a modified scale-free network with the power exponent $\alpha$ by applying a random removal of links with the survival rate $B(k)=\left(k / k_{\max }\right)^{\frac{\alpha-\beta}{\beta}}$. A link connecting to a node with in-degree $k$ is removed with a probability $1-B(k)$, and it remains with a probability $B(k)$. Moreover, as we mentioned in Sec. IV, our method can theoretically result in a scale-free network for any initial degree distribution. Our trimming method is a technique that derives a scale-free network from a given complex network by link reduction.

Next, let us discuss the application. The networks analyzed in this paper represent money flow and its conjugate-material or service flow-and drainage basin networks were extracted from both networks.

As an example of a global flow, we closely examine a cluster of a business network extracted using our method. Figure 7 is 


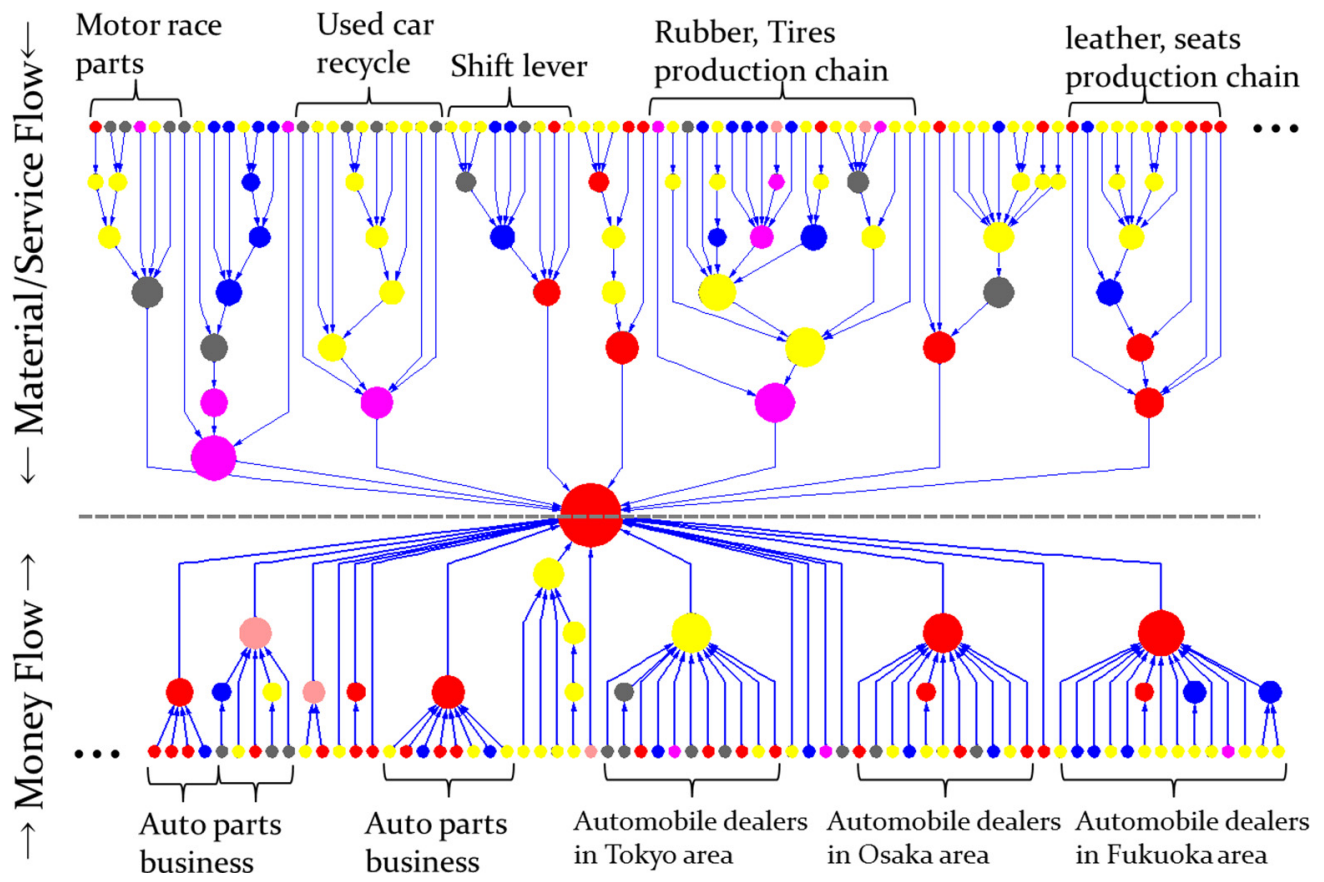

FIG. 7. (Color) Part of the treelike drainage basin structure flowing toward an automobile production company (the largest red square). The lower half of the figure is drawn by the money flow basin structure, and the upper half shows the drainage basin network of the material or service flow. The size of each node specifies its drainage basin size. Color denotes industry: red, yellow, gray, fuschia, blue, and pink denote manufacturing, retail trade, services, transportation and telecommunications, construction, and others, respectively.

an example of basin flows of money and materials or services to a major automobile production company. The trimming method clearly extracts the main structure of the supply-chain network of the material or service flow in the upper half. The tree structure indicates that a car is a composite of various parts, such as seats, rubber tires, and shift levers, made of raw materials coming from the peripheral nodes. In the lower half of the money-flow network, we find that the products are delivered to various areas in Japan and ultimately sold to consumers at the stores, represented by the lower peripheral nodes. We can also determine the sales chains for additional auto parts, such as navigation systems and audio sets.

Similarly, other clusters are obtained by making basin flows for materials or services and money toward major companies in various industries, including textiles, IT, precious metals, and medical devices.

As an application of this method to an interfirm trading network, we can regard the basin size of each node as a kind of indicator of the importance of network flow. Assume that a node is lost for some reason such as bankruptcy or disaster. Then money flow or material or service flow stops at the node, and the number of affected nodes can be approximated by the basin size as a rough approximation.

In the real network, such an estimation of flow change for removal of a node is very complicated as there are multiple links among nodes. As a generalization of the trimming procedure, we can consider trimming of third- or higherrank nodes among neighbors. Then we will have top- and second-rank links for each node, therefore many additional links will exist connecting the treelike basin structures defined for the top-rank nodes. The network topology will become much more complicated, but we may still be able to estimate the flow change in this less trimmed network. For example, if an additional link is connecting a pair of nodes in the same basin of the bankrupted node, then such a link will be inert. On the other hand, if a link connects different basins, then it will work as a bypass sustaining flows among nodes instead of the bankrupted node.

\section{CONCLUSION}

In conclusion, we proposed a method to extract a basin structure for any directed network by trimming all links except for the link to the largest neighbor. Our method provides values for the characterization of each node, basin degrees, and the drainage basin size, with respect to a node. These values characterize the flow topology, and we found similarities and differences between the money flow and the river flow.

We applied our method to a real interfirm trading network containing many loops as a natural characteristic. Our method removed these cyclic relations and extracted the hierarchical structure of the firms. The extracted tree structure exposes the accumulation structure of money or materials or services, which is a sequence of a number of firms managing from raw materials to completed products as a branch in the tree. The clusters appear to be roughly separated according to the category of products. This type of community extraction is not based on popular modularity maximization.

\section{ACKNOWLEDGMENTS}

This work was supported by a Grant-in-Aid for Scientific Research (B), Grant No. 26310207, a Grant-in-Aid for 
Scientific Research (C), Grant No. 24540395, and a Grantin-Aid for JSPS Fellows, Grant No. 26.11732. We thank the
Center for TDB Advanced Data Analysis and Modeling, Tokyo

Institute of Technology, for providing the datasets.
[1] J. D. Noh and H. Rieger, Phys. Rev. Lett. 92, 118701 (2004).

[2] S. Brin and L. Page, Comput. Netw. ISDN Syst. 30, 107 (1998).

[3] A. Fronczak and P. Fronczak, Phys. Rev. E 80, 016107 (2009).

[4] A. Fronczak and P. Fronczak, Phys. Rev. E 74, 026121 (2006).

[5] A. Baronchelli and R. Pastor-Satorras, Phys. Rev. E 82, 01111 (2010).

[6] B. Tadić, G. J. Rodgers, and S. Thurner, Int. J. Bifurcation Chaos 17, 2363 (2007).

[7] H. Watanabe, H. Takayasu, and M. Takayasu, New J. Phys. 14, 043034 (2012).

[8] D. Card, J. Labor Econ. 19, 22 (2001).

[9] F. Simini, M. C. González, A. Maritan, and A.-L. Barabási, Nature (London) 484, 96 (2012).

[10] R. Piermartini and L. Rousova, WTO Staff Working Paper, ERSD-2008-06 (WTO, Geneva, 2008).

[11] K. Tamura, W. Miura, H. Takayasu, S. Kitajima, H. Goto, and M. Takayasu, Int. J. Mod. Phys. Conf. Ser. 16, 93 (2012).

[12] A. E. Scheidegger, Int. Assoc. Sci. Hydrol. Bull. 12, 57 (1967).

[13] H. Takayasu and H. Inaoka, Phys. Rev. Lett. 68, 966 (1992).

[14] R. E. Horton, Geol. Soc. Am. Bull. 56, 275 (1945).

[15] A. N. Strahler, Geol. Soc. Am. Bull. 63, 1117 (1952).

[16] A. N. Strahler, Trans. Am. Geophys. Union 8, 913 (1957).

[17] R. L. Shreve, J. Geol. 74, 17 (1966).

[18] E. Van Bavel and J. A. Spaan, Circ. Res. 71, 1200 (1992).

[19] K. Horsfield, Circ. Res. 42, 593 (1978).
[20] L. B. Leopold, J. Theor. Biol. 31, 339 (1971).

[21] R. L. Shreve, J. Geol. 75, 178 (1967).

[22] P. La Barbera, Water Resourc. Res. 25, 735 (1989).

[23] D. G. Tarboton, R. L. Bras, and I. Rodriguez-Iturbe, Water Resourc. Res. 24, 1317 (1988).

[24] R. Feenstra, Advanced International Trade: Theory and Evidence (Princeton University Press, Princeton, NJ, 2003).

[25] M. Takayasu, S. Sameshima, T. Ohnishi, Y. Ikeda, H. Takayasu, and K. Watanabe, Ann. Rep. of the Earth Simulator Center, April 2007-March 2008 (2008), Vol. 263.

[26] T. Ohnishi, H. Takayasu, and M. Takayasu, Progr. Theor. Phys. Suppl. 179, 157 (2009).

[27] W. Miura, H. Takayasu, and M. Takayasu, Phys. Rev. Lett. 108, 168701 (2012).

[28] J. E. Anderson, Am. Econ. Rev. 69, 106 (1979).

[29] R. N. Mantegna, Eur. Phys. J. B 11, 193 (1999).

[30] J. B. Glattfelder and S. Battiston, Phys. Rev. E 80, 036104 (2009).

[31] K. Anand, B. Craig, and Goetz von Peter, Quantitat. Fin. 15, 625 (2015).

[32] H. Watanabe, H. Takayasu, and M. Takayasu, Physica A 392, 741 (2013).

[33] M. Takayasu, K. Tamura, A. Yougai, H. Goto, N. Sakai, W. Miura, I. Ono, and H. Takayasu (unpublished).

[34] M. E. J. Newman, SIAM Rev. 45, 167 (2003). 